\title{
KLRD1-expressing natural killer cells predict influenza susceptibility
}

Erika Bongen ${ }^{1,2} \mathbb{D}$, Francesco Vallania ${ }^{1,3}$, Paul J. Utz ${ }^{1,2,4}$ and Purvesh Khatri ${ }^{1,2,3^{*}}$

\begin{abstract}
Background: Influenza infects tens of millions of people every year in the USA. Other than notable risk groups, such as children and the elderly, it is difficult to predict what subpopulations are at higher risk of infection. Viral challenge studies, where healthy human volunteers are inoculated with live influenza virus, provide a unique opportunity to study infection susceptibility. Biomarkers predicting influenza susceptibility would be useful for identifying risk groups and designing vaccines.

Methods: We applied cell mixture deconvolution to estimate immune cell proportions from whole blood transcriptome data in four independent influenza challenge studies. We compared immune cell proportions in the blood between symptomatic shedders and asymptomatic nonshedders across three discovery cohorts prior to influenza inoculation and tested results in a held-out validation challenge cohort.
\end{abstract}

Results: Natural killer (NK) cells were significantly lower in symptomatic shedders at baseline in both discovery and validation cohorts. Hematopoietic stem and progenitor cells (HSPCS) were higher in symptomatic shedders at baseline in discovery cohorts. Although the HSPCs were higher in symptomatic shedders in the validation cohort, the increase was statistically nonsignificant. We observed that a gene associated with NK cells, KLRD1, which encodes CD94, was expressed at lower levels in symptomatic shedders at baseline in discovery and validation cohorts. KLRD1 expression in the blood at baseline negatively correlated with influenza infection symptom severity. KLRD1 expression $8 \mathrm{~h}$ postinfection in the nasal epithelium from a rhinovirus challenge study also negatively correlated with symptom severity.

Conclusions: We identified KLRD1-expressing NK cells as a potential biomarker for influenza susceptibility. Expression of KLRD1 was inversely correlated with symptom severity. Our results support a model where an early response by KLRD1-expressing NK cells may control influenza infection.

Keywords: Influenza, Natural killer cells, Hematopoietic stem and progenitor cells, KLRD1, CD94

\section{Background}

Influenza is a major public health problem that causes 9 to 35 million illnesses annually in the USA [1]. Children, older adults, pregnant women, and immunocompromised patients are at an increased risk of influenza infection. Within healthy young adults, influenza susceptibility is difficult to predict as responses to influenza exposure vary from no detectable infection to severe disease. A better understanding of the immune determinants of influenza susceptibility is necessary to

\footnotetext{
* Correspondence: pkhatri@stanford.edu

${ }^{1}$ Institute for Immunity, Transplantation and Infection, Stanford University

School of Medicine, Stanford, CA 94305, USA

${ }^{2}$ Program in Immunology, Stanford University School of Medicine, Stanford

94305, CA, USA

Full list of author information is available at the end of the article
}

identify novel high-risk populations and design better vaccines.

Human influenza challenge studies provide a unique opportunity to study influenza susceptibility. In these studies, healthy individuals are inoculated with live influenza virus, and viral shedding titers and self-reported symptom scores are measured over the course of infection. Infected individuals fall into four groups: symptomatic shedders, asymptomatic nonshedders, symptomatic nonshedders, and asymptomatic shedders. Previous challenge studies have used transcriptional data to distinguish symptomatic shedders from asymptomatic nonshedders post-infection [2], detect infection prior to symptom onset [3], develop transcriptional signatures of symptom status $[4,5]$, and prototype individualized

(c) The Author(s). 2018 Open Access This article is distributed under the terms of the Creative Commons Attribution 4.0 International License (http://creativecommons.org/licenses/by/4.0/), which permits unrestricted use, distribution, and 
predictors for infection [6]. However, to our knowledge, no cellular or transcriptional signatures that can predict infection susceptibility prior to inoculation have been reported.

Relatively little work has been done examining how preexisting immune cell populations affect influenza susceptibility. Wilkinson et al. demonstrated in an H3N2 influenza challenge study that higher baseline levels of influenza-specific CD4+ T cells in the blood were associated with reduced viral shedding and less severe symptoms [7]. Sridhar et al. followed healthy adults during two consecutive flu seasons and found that adults with higher baseline levels of influenza-specific CD8+ T cells experienced lower symptom severity [8]. To our knowledge, the role of immune cell frequencies in influenza susceptibility beyond the $\mathrm{T}$ cell compartment has not been described.

Cell mixture deconvolution is an established computational approach to estimate immune cell proportions from bulk tissue gene expression data, either from blood or solid tissue [9]. The key assumption of cell mixture deconvolution is that the gene expression of a bulk tissue sample can be explained by the underlying ratio of cell types and the expression profiles of those cell types. Deconvolution methods define specific cell types using a reference matrix, known as a basis matrix, of expected cell type expression. The basis matrix is used by an algorithm, such as linear regression, to predict the proportion of each cell type in bulk tissue samples. Cell mixture deconvolution has been used to profile the immune response to leprosy and across cancers $[10,11]$. We have described a deconvolution basis matrix, immunoStates, that accurately estimates cellular proportions for 20 immune cell subsets by reducing biological, methodological, and technical biases [12]. In this study, we used the immunoStates basis matrix with a linear regression model.

We hypothesized that immune cell populations at baseline (i.e., prior to exposure to influenza) may affect influenza susceptibility. To test this hypothesis, we used 4 influenza challenge studies (3 discovery, 1 validation) composed of 52 samples (40 discovery, 12 validation). We estimated proportions of 20 immune cell subsets in each sample using the immunoStates matrix and a linear regression model. We performed a multi-cohort analysis of estimated immune cell proportions between symptomatic shedders and asymptomatic nonshedders at baseline across the three discovery influenza challenge studies. Symptomatic shedders had lower proportions of natural killer (NK) cells at baseline in discovery cohorts and the held-out validation cohort. Symptomatic shedders had significantly higher proportions of hematopoietic stem and progenitor cells (HSPCs) at baseline. Although the validation cohort demonstrated the same trend, it was not statistically significant. NK cell-associated gene $K L R D 1$ expression was also significantly lower in the blood of symptomatic shedders at baseline in discovery and validation cohorts and correlated negatively with symptom severity. Increased KLRD1 expression may be associated with increased proportions of cytotoxic cells, as KLRD1 expression at baseline correlated with cytotoxic granule-associated genes CCL5, perforin (PRF1), and several granzymes (GZMA, GZMB, and GZMH). We also observed that KLRD1 expression decreased in the blood during the first $48 \mathrm{~h}$ of influenza infection. We examined KLRD1 expression in the nasal epithelium in human rhinovirus (HRV) and respiratory syncytial virus (RSV) infection as robust common immune response across these viruses has been described [13]. KLRD1 expression significantly increased in nasal epithelium during infection with HRV or RSV. In an HRV challenge cohort, symptom severity correlated negatively with expression of KLRD1 in the nasal epithelium $8 \mathrm{~h}$ post-infection. This data supports a model where a rapid antiviral response by KLRD1-expressing NK cells may control viral infection.

\section{Methods}

\section{Identification and preprocessing of cohorts}

We identified 4 influenza challenge studies consisting of 52 whole blood samples from the NCBI database Gene Expression Omnibus (GEO) (Table 1). We supplemented the influenza challenge cohorts with 7 acute viral infection studies consisting of 16 cohorts of 771 whole blood, PBMC, and nasal epithelium samples from GEO (Table 2) [14]. We excluded challenge studies with less than five asymptomatic nonshedders or five symptomatic shedders. We used phenotypic labels as reported by

Table 1 Influenza challenge cohorts

\begin{tabular}{llllllll}
\hline Cohort & Group & Virus & Tissue & $\begin{array}{l}\text { Asymptomatic } \\
\text { nonshedders }\end{array}$ & $\begin{array}{l}\text { Symptomatic } \\
\text { shedders }\end{array}$ & Platform & Citations \\
\hline GSE73072 challenge A & Discovery challenge & H1N1 & Whole blood & 6 & 8 & Affymetrix & {$[2,3,6]$} \\
GSE73072 challenge B & Discovery challenge & H3N2 & Whole blood & 6 & 7 & Affymetrix & {$[2,3,6]$} \\
GSE73072 challenge C & Discovery challenge & H3N2 & Whole blood & 6 & 7 & Affymetrix & {$[6]$} \\
GSE61754 & Validation challenge & H3N2 & Whole blood & 5 & 7 & Illumina & {$[5]$} \\
Total & & 2 & 1 & 23 & 29 & 2 & \\
\hline
\end{tabular}


Table 2 Additional viral infection cohorts

\begin{tabular}{|c|c|c|c|c|c|c|}
\hline Cohort & Virus & Tissue & Controls & Acute infection & Platform & Citations \\
\hline GSE11348 & HRV & Nasal scrapings & 15 & 15 & Affymetrix & [22] \\
\hline GSE97742 HRV & HRV & Nasopharyngeal swabs & 30 & 30 & Illumina & [23] \\
\hline GSE97742 RSV & RSV & Nasopharyngeal swabs & 38 & 38 & Illumina & [23] \\
\hline GSE97742 RSVCO & $\begin{array}{l}\text { RSV co-infected with } \\
\text { other viruses }\end{array}$ & Nasopharyngeal swabs & 15 & 15 & Illumina & [23] \\
\hline GSE61821 mild H1N1 & Seasonal H1N1 & Whole blood & 36 & 32 & Illumina & {$[40]$} \\
\hline GSE61821 mild H3N2 & Seasonal H3N2 & Whole blood & 13 & 15 & Illumina & {$[40]$} \\
\hline GSE61821 severe H1N1 & Seasonal H1N1 & Whole blood & 19 & 16 & Illumina & {$[40]$} \\
\hline GSE61821 severe H3N2 & Seasonal H3N2 & Whole blood & 6 & 7 & Illumina & {$[40]$} \\
\hline GSE61821 pandemic H1N1 & Pandemic H1N1 & Whole blood & 8 & 10 & Illumina & {$[40]$} \\
\hline GSE68310 flu & Influenza A & Whole blood & 40 & 34 & Illumina & [24] \\
\hline GSE43777 & Dengue & PBMC & 45 & 45 & Affymetrix & [41] \\
\hline GSE51808 & Dengue & Whole blood & 9 & 13 & Affymetrix & {$[42]$} \\
\hline GSE68310 HRV & HRV & Whole blood & 20 & 20 & Illumina & [24] \\
\hline GSE97741 HRV & HRV & Whole blood & 25 & 24 & Illumina & [23] \\
\hline GSE67059 RSV & RSV & Whole blood & 20 & 65 & Illumina & [43] \\
\hline GSE97741 RSV & RSV & Whole blood & 25 & 28 & Illumina & [23] \\
\hline Total & 8 & 4 & 364 & 407 & & \\
\hline
\end{tabular}

the original authors. All datasets used were publicly available (Additional file 1: Supplemental Methods).

\section{Cell mixture deconvolution using immunoStates}

We performed cell mixture deconvolution using the immunoStates basis matrix and a linear regression model, as described previously, to estimate the immune cell frequencies for 20 immune cell subsets in blood or nasal epithelium gene expression data [12]. We removed all cell types that were not detected in any samples (Additional file 1: Table S1). If a cell type was detected in a subset of samples, values of zero were set to an arbitrarily low number and each sample was rescaled so that the cell type proportions summed to $100 \%$ in each sample.

\section{Integrated multi-cohort analysis of cellular proportions}

We performed an integrated multi-cohort analysis using the MetaIntegrator $R$ package [15]. To analyze differences in cell proportions, we utilized random effects inverse variance model-based meta-analysis by combining effect sizes, as described previously $[13,15,16]$. We estimated the change in proportion for each cell type in each cohort between symptomatic shedders and asymptomatic nonshedders as Hedge's adjusted $g$. We combined the changes in cellular proportion for each cell type into a summary effect size using a linear combination of study-specific effect sizes, where each cohort-specific effect size was weighted by the inverse of that cohort's pooled variance $[15,17]$. We performed multiple hypotheses testing correction using the Benjamini-Hochberg false discovery rate (FDR) [18].

\section{Results}

\section{Dataset description}

We identified four human influenza challenge studies from the NCBI database Gene Expression Omnibus (GEO) (Table 1 and Fig. 1) [14]. Each of these studies profiled the whole blood transcriptome of healthy individuals inoculated with live $\mathrm{H} 1 \mathrm{~N} 1$ or $\mathrm{H} 3 \mathrm{~N} 2$ influenza at baseline and the subsequent 2-7 days. These studies defined viral shedding status based on influenza laboratory tests and symptom status based on self-reported modified Jackson scores $[5,6]$.

We chose three of the challenge cohorts as discovery cohorts as they were part of a single study and all profiled samples using Affymetrix microarrays [6]. The remaining dataset, GSE61754, profiled samples using Illumina microarrays and was used as a validation cohort [5]. This choice allowed us to ensure that our deconvolution analysis was robust to the microarray platform used. We only included baseline samples from subjects with concordant symptom and shedding status (symptomatic shedders and asymptomatic nonshedders).

\section{Integrated multi-cohort analysis of estimated cell proportions}

We hypothesized that the immune cell profiles of symptomatic shedders and asymptomatic nonshedders would be different prior to inoculation. To test this hypothesis, 


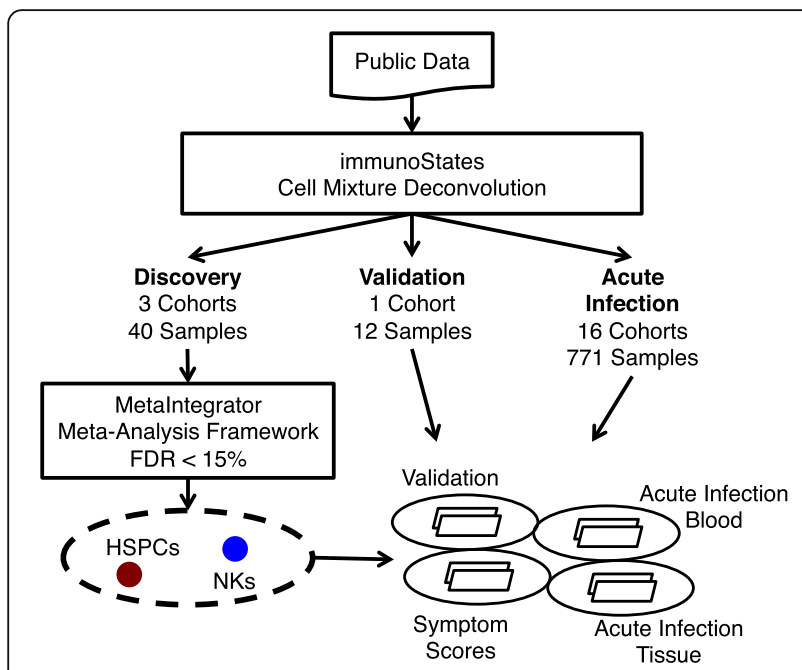

Fig. 1 Schematic of experimental design. From NCBI GEO, we identified gene expression microarray datasets from influenza viral challenge studies and studies of naturally acquired infection. Using immunoStates cell mixture deconvolution, we estimated the immune cell proportions in each sample. We separated the challenge studies into discovery and validation cohorts. We analyzed differences at baseline between symptomatic shedders and asymptomatic nonshedders using Metalntegrator, a meta-analysis framework. We identified immune cell types with significantly different proportions between symptomatic shedders and asymptomatic nonshedders, which we further investigated in an influenza challenge validation cohort, and cohorts of naturally acquired acute infections

we estimated proportions of 20 immune cell types in each sample in each cohort using immunoStates and a linear regression model [12]. We removed 8 out of 20 cell types from further analysis as they were not detected in at least one dataset (Additional file 1: Table S1). A multi-cohort analysis of estimated cellular proportions for the remaining cell types in discovery cohorts using MetaIntegrator found that proportions of NK cells were significantly lower $(P=0.012$, FDR $<15 \%$; Fig. $2 a)$, and hematopoietic stem and progenitor cells (HSPCs) were significantly higher $(P=0.017$, FDR $<15 \%$; Fig. $2 \mathrm{~b})$ in symptomatic shedders at baseline. We also observed significantly lower NK cell proportions at baseline in symptomatic shedders in the validation cohort $(P=$ 0.045; Fig. 2c). Although the validation cohort exhibited a trend of higher proportions of HSPCs in symptomatic shedders at baseline, this increase was not statistically significant $(P=0.13$; Fig. $2 \mathrm{~d})$.

\section{Identification of KLRD1 as an NK cell-associated gene relevant to influenza challenge}

A basis matrix in deconvolution defines a set of genes as a proxy for the presence of a cell type in a sample. Therefore, a significant reduction in NK cell proportions suggests that a subset of genes in immunoStates representing NK cells should be downregulated at baseline in symptomatic shedders compared to asymptomatic nonshedders. One of the 19 NK cell-related genes in immunoStates, KLRD1, was significantly downregulated in symptomatic shedders in discovery cohorts (summary $\mathrm{ES}=-0.54, P=0.026$; Fig. 3a) and the validation cohort $(P=3.3 \mathrm{e}-3$; Fig. $3 \mathrm{~b})$. In a validation cohort, KLRD1 expression in the blood prior to infection differentiated between symptomatic shedders and asymptomatic nonshedders with high accuracy (AUROC $=0.91,95 \% \mathrm{CI}$ 0.75-1.0; Fig. 3c). Interestingly, the baseline expression of $K L R D 1$ was significantly inversely correlated with total symptom scores $(r=-0.79, P=5.2 \mathrm{e}-4$; Fig. $3 \mathrm{~d})$ in the validation cohort and was marginally significant $(r=-0.48, P=0.07)$ in one of the two discovery cohorts where total symptom scores were available (Additional file 1: Figure S1). This suggests that KLRD1-expressing NK cells may be important for controlling influenza symptom severity.

\section{KLRD1 baseline expression correlates with $K L R C 3$ and cytotoxic granule associated genes}

KLRD1 encodes NK cell receptor CD94 that forms a heterodimer with several NKG2 family members [19]. To determine whether KLRD1 expression was associated with a particular NKG2 family member, we correlated KLRD1 expression at baseline with three NKG2 family member encoding genes: KLRC1, KLRC2, and KLRC3. Only KLRC3, which encodes protein isoforms NKG2E and NKG2H, significantly correlated with KLRD1 in the validation cohort $(r=0.75, \quad P=1.3 \mathrm{e}-3$; Fig. $4 \mathrm{a})$ and discovery cohorts $(r=0.4, P=7.1 \mathrm{e}-3$; Additional file 1 : Figure S2a).

To determine whether expression of KLRD1 was associated with a cytotoxic transcriptional signature, we correlated expression of KLRD1 at baseline with genes associated with cytotoxic granules. While releasing cytotoxic granules, NK cells also release CCL5 [20]. CCL5 expression positively correlated with $K L R D 1$ in validation $(r=0.78, P=6 \mathrm{e}-4$; Fig. $4 \mathrm{~b})$ and discovery cohorts $(r=0.74$, $P=7.3 \mathrm{e}-9$; Additional file 1: Figure S2b). Perforin (PRF1) and granzymes (GZMA, GZMB, GZMH) are critical components of cytotoxic granules secreted by NK cells to kill target cells [21]. Expression of each cytotoxic granule gene was positively correlated with KLRD1 expression at baseline in the validation cohort $(0.57 \leq r \leq 0.62, P<0.03$; Fig. $4 \mathrm{c}-\mathrm{f})$ and in the discovery cohorts $(0.76 \leq r \leq 0.83$, $P<3$ e-9; Additional file 1: Figure S2c-f).

\section{KLRD1 expression decreases in the blood and increases in} the nasal epithelium after respiratory viral infection

KLRD1 expression further decreased in the blood within the first $48 \mathrm{~h}$ of infection in both the discovery (Fig. 5a) and validation (Fig. $5 \mathrm{~b}$ ) cohorts. One possibility for the reduction in KLRD1 expression in the blood following 
a

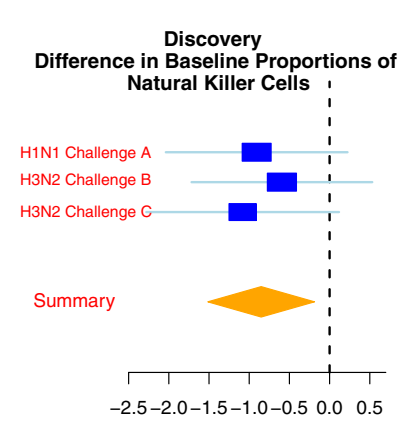

Standardized Mean Difference (log2 scale)

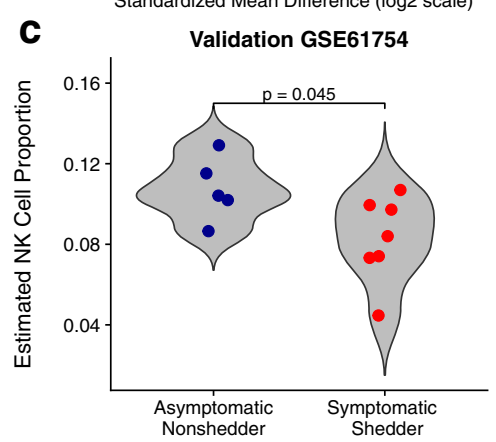

b

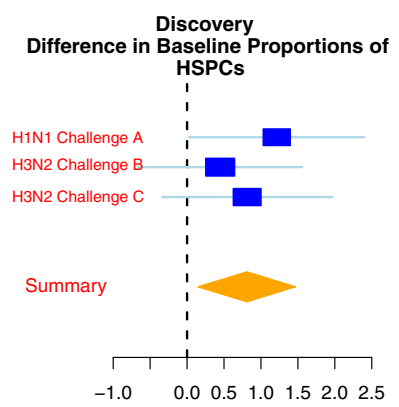

Standardized Mean Difference (log2 scale)

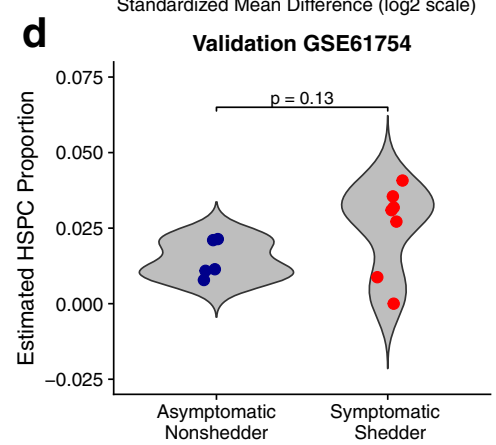

Fig. 2 Differences in estimated cell type proportions between asymptomatic nonshedders and symptomatic shedders before infection. Immune cell proportions were estimated at baseline using cell mixture deconvolution. Forest plots of effect sizes of a NK cells (effect size $=-0.85, P=0.012$ ) and b HSPCs (effect size $=0.81, P=0.017$ ) in discovery cohorts. Positive effect sizes indicate higher levels while negative effect sizes indicate lower levels for that cell type in symptomatic shedders. The $x$ axes represent standardized mean difference between symptomatic shedders and asymptomatic nonshedders, computed as Hedges' $g$, in log2 scale. The size of the blue rectangles is proportional to the SEM difference in the study. Whiskers represent the $95 \%$ confidence interval. The yellow diamonds represent overall, combined mean difference for a given cell type. Width of the yellow diamonds represents the $95 \%$ confidence interval of overall mean difference. Violin plots of estimated cell proportions of $\mathbf{c}$ NK cells (effect size $=-1.18, P=0.045)$ and $\mathbf{d}$ HSPCS (effect size $=0.79, P=0.13$ ) at baseline in validation cohort GSE61754. NK, natural killer. HSPC, hematopoietic stem and progenitor cells

infection is that KLRD1-expressing NK cells are trafficking to the site of infection. Therefore, we sought to examine expression of KLRD1 in nasal epithelium during acute influenza infection. However, no publicly available studies to our knowledge have profiled human nasal epithelium expression during influenza infection. We have previously described a robust common host immune response to acute respiratory viral infection including influenza, human rhinovirus (HRV), and respiratory syncytial virus (RSV) [13]. Therefore, we utilized a HRV challenge study (GSE11348), and a cohort of children naturally infected with HRV, RSV, or RSV co-infected with other pathogens (RSVco) (GSE97742) [22, 23]. KLRD1 was expressed at significantly higher levels in virally infected nasal epithelium samples (effect size $=0.77, P=0.0011$; Fig. $5 \mathrm{c}$ ).

In the HRV challenge study (GSE11348), KLRD1 expression at $8 \mathrm{~h}$ post-infection was significantly inversely correlated with symptom severity $(r=-0.6, P=0.031$; Fig. 5d) similar to influenza challenge studies. We also observed significant positive correlations between KLRD1 expression and expression of KLRC3 $(r=0.82$, $P=6.5 \mathrm{e}-4$, Fig. 5e) and HLA-E $(r=0.76, p=0.0028$,
Fig. 5f). This data suggests a model where a rapid response by KLRD1- and KLRC3-expressing NK cells with concurrent upregulation of $H L A-E$ by the surrounding tissue may reduce viral infection severity.

\section{HSPCs decrease in the blood during naturally acquired viral infections}

Although the difference in HSPC proportions was not statistically significant in validation cohort GSE61754, we observed a trend for higher proportions of HSPCs in symptomatic shedders at baseline (effect size $=0.79, P=$ 0.13; Fig. 2d). It was surprising that HSPCs demonstrated any association with influenza susceptibility, as very little is known about the role of circulating HSPCs in acute infection, particularly in humans. Thus, we investigated changes in HSPC proportions in the blood during acute viral infection. We extended our analysis by performing a meta-analysis of estimated HSPC proportions from naturally acquired influenza cohorts with 236 samples. Individuals with acute influenza infection had consistently lower proportions of HSPCs in the blood than the control time point (summary effect size $=-2.0, P<1 \mathrm{e}-13$; Fig. 6a). To determine whether this 

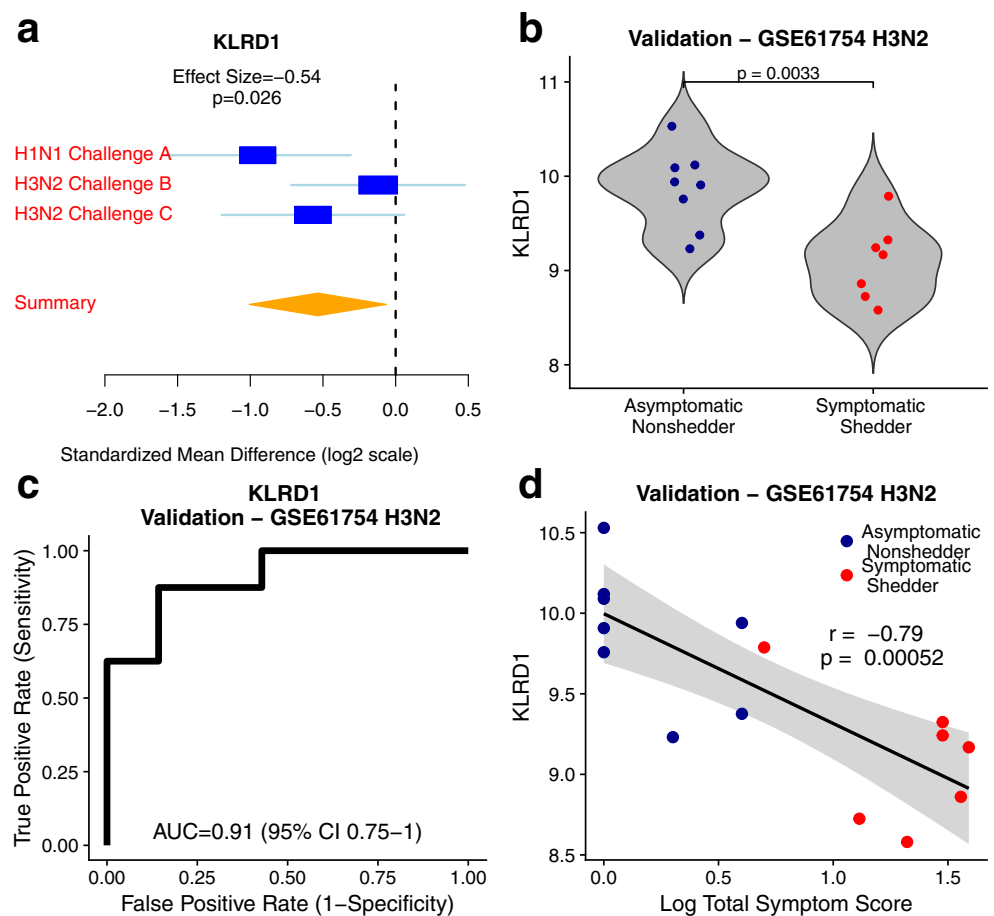

Fig. 3 CD94 encoding gene KLRD1 is differentially expressed between asymptomatic nonshedders and symptomatic shedders and correlates with symptom severity at baseline. a Forest plot of effect sizes of baseline KLRD1 expression in discovery cohorts (summary effect size $=-0.54, P=0.026$ ). The $x$ axes represent standardized mean difference between symptomatic shedders and asymptomatic nonshedders, computed as Hedges' $g$, in log 2 scale. The size of the blue rectangles is proportional to the SEM difference in the study. Whiskers represent the 95\% confidence interval. The yellow diamonds represent overall, combined mean difference for a given gene. Width of the yellow diamonds represents the $95 \%$ confidence interval of overall mean difference. $\mathbf{b}$ Violin plot of KLRD1 expression at baseline in validation cohort GSE61754 ( $P=0.0033)$. $\mathbf{c}$ ROC plot of performance of KLRD1 expression to differentiate asymptomatic nonshedders and symptomatic shedders at baseline ( $A U C=0.91,95 \% \mathrm{Cl} 0.75-1.0$ ). $\mathbf{d}$ Correlation between baseline KLRD1 expression and logged total symptom score in validation cohort GSE61754 $(r=-0.79, p=0.00052)$

was influenza-specific, we performed a meta-analysis of estimated HSPC proportions from naturally occurring non-influenza acute viral cohorts. We included six cohorts of acute dengue, HRV, and RSV infection (339 samples total). We observed a significant decrease in HSPC proportions in acute non-influenza viral infection (effect size $=0.5, P<0.001$; Fig. 6b).

We further investigated the dynamics of changes in HSPC proportions in the blood during influenza infection using GSE68310, where individuals provided a baseline healthy sample at the beginning of the flu season, and returned to the clinic within $48 \mathrm{~h}$ of symptom onset (day 0) [24]. We observed a significant decrease in HSPC proportions $(p<0.0001$; Fig. 6c). This decrease in HSPC proportions continued through day 6 , and HSPC proportions returned to baseline levels by day 21 (Fig. 6c).

\section{HSPC proportions decrease in nasal epithelium over the course of rhinovirus challenge and correlate with increases in $\mathrm{MDC}$ and $\mathrm{M} 1$ macrophage proportions}

To study the presence of HSPCs at the site of infection, we examined HSPC proportions from nasal scrapings of human volunteers inoculated with HRV (GSE11348) [22]. HSPC proportions sharply decreased $48 \mathrm{~h}$ post-infection in nasal scrapings $(P=1.3 \mathrm{e}-5$; Fig. $6 \mathrm{~d})$. This decrease could result from trafficking, cell death, or differentiation of HSPCs into mature myeloid cells. To test the hypothesis that HSPCs differentiate into mature cells during viral infection, we correlated the changes in HSPC proportions with the changes of myeloid dendritic cell $(\mathrm{mDC})$ and $\mathrm{M} 1$ macrophage proportions between pre-infection and $48 \mathrm{~h}$ post-infection. Reductions of HSPC proportions strongly correlated with increased proportions of M1 macrophages $(r=-0.84, p=9.3 \mathrm{e}-5$; Fig. 6e) and mDCs $(r=-0.84, P=8.5 e-5$; Fig. 6 f), both of which derive from the hematopoietic lineage. This finding is supported by data derived from a cohort of children acutely infected with HRV, RSV, or a co-infection of RSV and other pathogens [23]. We observed in this additional cohort that proportions of HSPCs during acute infection from nasopharyngeal swabs negatively correlated with proportions of M1 macrophages and $\mathrm{mDCs}(-0.82<r<-0.22 ; 2 \mathrm{e}-4<p<0.24$; Additional file 1: Figure S3). As the samples with the lowest proportions of HSPCs were the samples with 

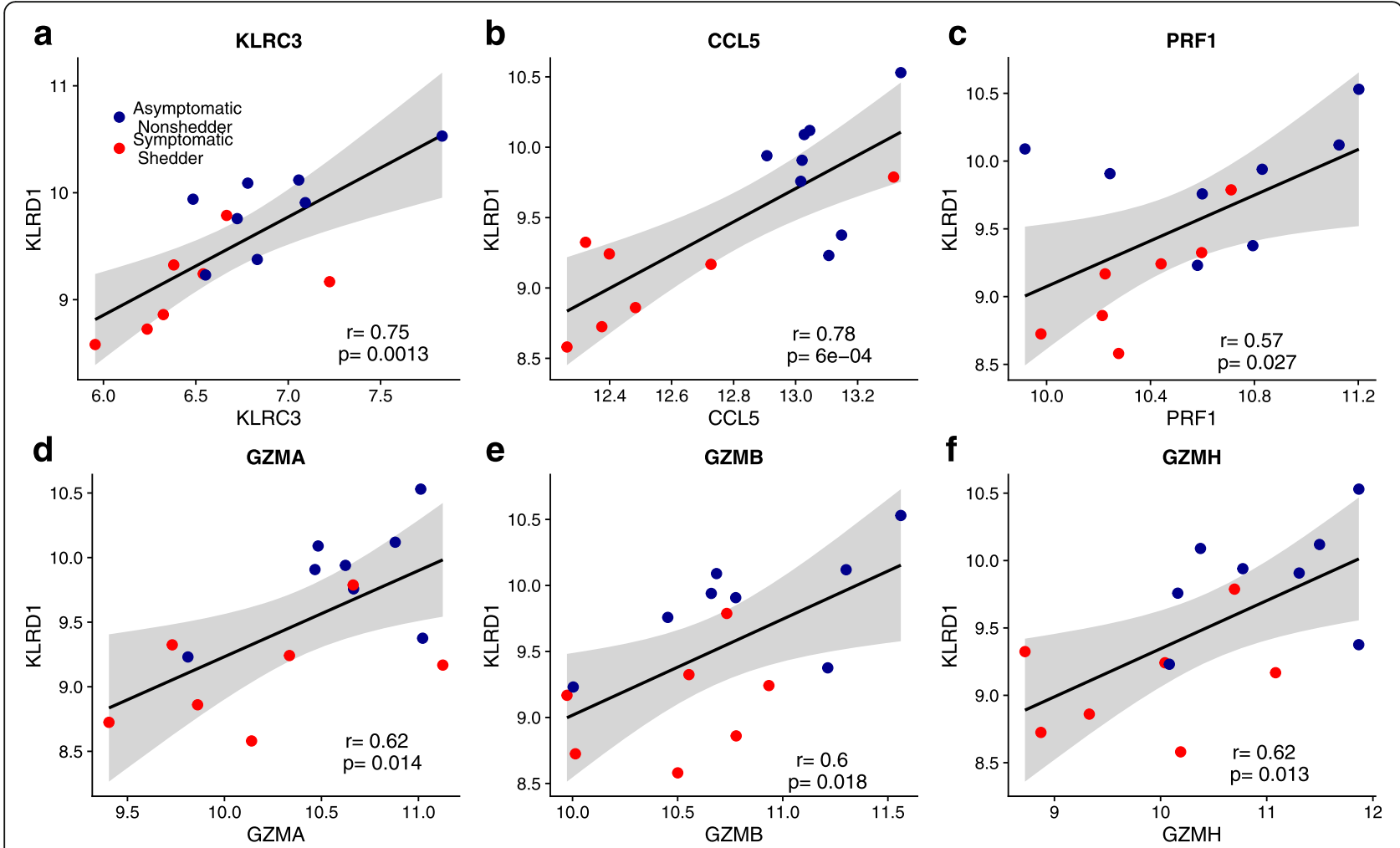

Fig. $4 K L R D 1$ correlates with $K L R C 3$, and cytotoxic granule-associated genes before infection. Gene expression from validation cohort GSE61754 prior to infection demonstrating correlations between $K L R D 1$ expression and $\mathbf{a} K L R C 3(r=0.75, P=0.0013)$ and $\mathbf{b}$ - $\mathbf{f}$ cytotoxic granule-associated genes: CCL5 $(r=0.78, P=0.0006)$, perforin (PRF1, $r=0.57, P=0.027)$, granzyme A $(G Z M A, r=0.62, P=0.014)$, granzyme $B(G Z M B, r=0.6, P=0.018)$, and granzyme $\mathrm{H}(G Z M H, r=0.62, P=0.013)$

the highest proportions of M1 macrophages and mDCs, this supports a model where HSPCs differentiate into M1 macrophages and $\mathrm{mDCs}$ at the site of infection in humans.

\section{Discussion}

Here, we tested a hypothesis that the baseline immune profile prior to influenza inoculation can predict which subject will become infected. We applied cell mixture deconvolution of whole blood transcriptome profiles from four independent influenza challenge studies. Symptomatic shedders had lower NK cell proportions prior to influenza inoculation both in discovery and validation cohorts. Symptomatic shedders had significantly higher HSPC proportions in discovery cohorts with a statistically non-significant trend in the validation cohort. NK cell-associated gene KLRD1 (CD94) was expressed in the blood at lower levels in symptomatic shedders at baseline in both discovery and validation cohorts, which likely reflects differences in NK cell proportions as KLRD1 was one of the genes used in immunoStates for estimating proportions of NK cells. Baseline KLRD1 levels negatively correlated with symptom severity and positively correlated with expression of cytotoxic granule-associated genes. Our results support a model where a rapid response by KLRD1-expressing NK cells can lessen severity of or may prevent influenza infection.

NK cells are innate immune cells that can recognize and lyse malignant or virally infected cells [28]. NK cells express a variety of activating and inhibitory receptors that lead to a diverse pool of NK cell phenotypes [26]. KLRD1 encodes NK cell receptor CD94, which forms a heterodimer with an NKG2 family member, and recognizes HLA-E on target cells [26]. Whether the CD94/ NKG2 complex is activating or inhibitory depends on the NKG2 family member involved. The NKG2 family includes inhibitory receptors NKG2A and NKG2B, activating receptor $\mathrm{NKG} 2 \mathrm{C}$, and poorly understood members NKG2E and NKG2H [19, 25]. NKG2E is not expressed on NK cell surface [26], whereas NKG2H is expressed on the surface of a small fraction of human NK cells [27]. By surveying HLA-E levels on target cells, the CD94/NKG2 complex is thought to detect general downregulation of HLA complexes by viruses or cancer [19].

The CD94/NKG2E receptor complex has been shown to be essential for mouse survival when exposed to mousepox [28]. On the other hand, CD94-deficient mice are not susceptible to mouse cytomegalovirus, 


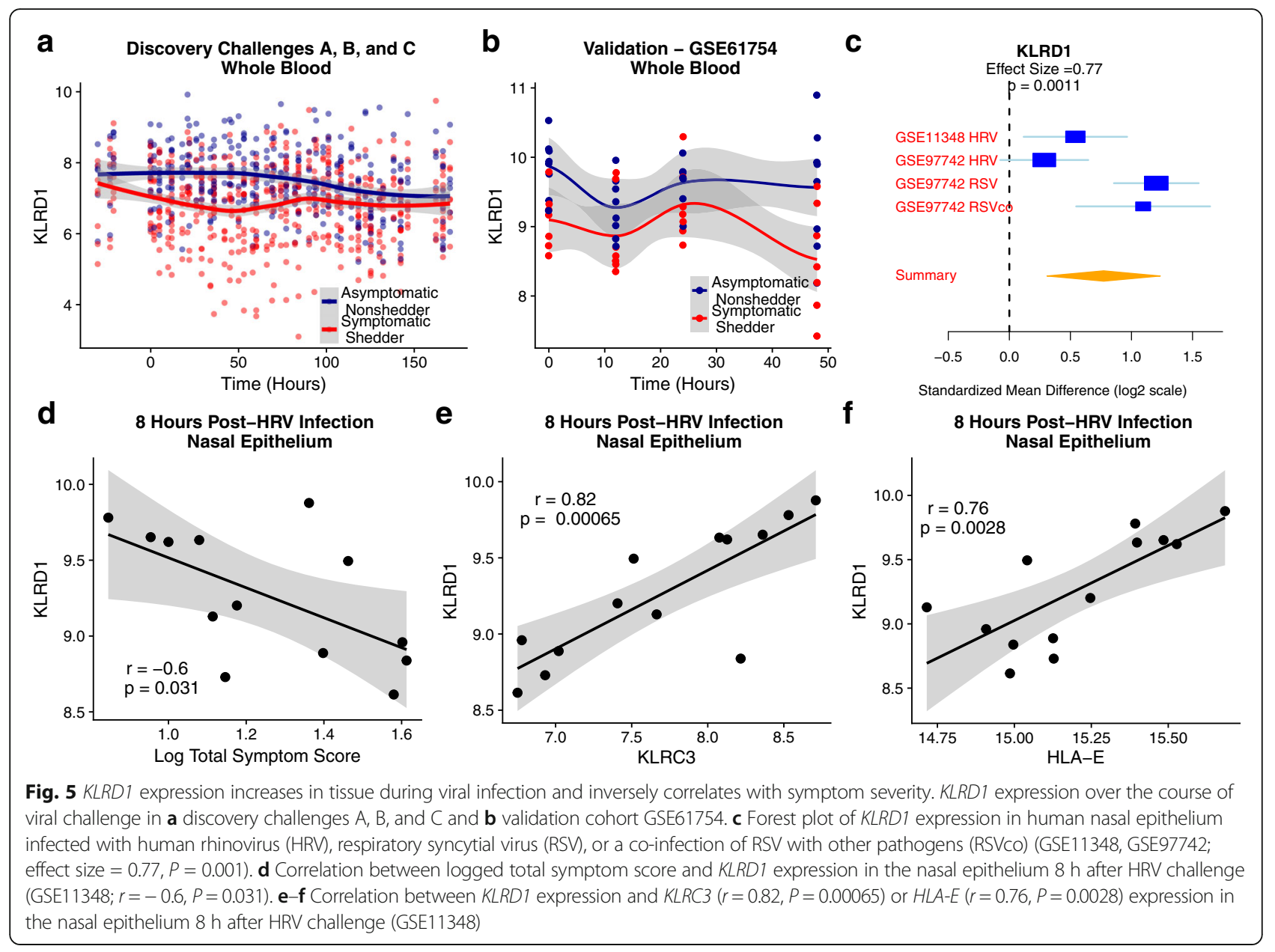

lymphocytic choriomeningitis virus, vaccinia virus, Listeria monocytogenes, or lethal influenza challenge [29, 30]. Importantly, mouse studies have shown that NK cells are harmful upon lethal challenge by promoting excessive lung inflammation, but beneficial during sublethal influenza challenge by promoting the antiviral immune response [21, 31, 32]. These observations in mouse studies further support our results as human challenge studies are most similar to sublethal mouse influenza models.

We observed that KLRD1 expression in the blood is downregulated in symptomatic shedders at baseline and inversely correlated with symptom severity in a validation cohort. As KLRD1 expression in the blood reflects NK cell numbers, this suggests that KLRD1-expressing NK cells are protective against influenza infection in humans. Furthermore, KLRD1 expression in the blood correlated with expression of cytotoxic granule-associated genes: CCL5, perforin (PRF1), and several granzymes (GZMA, GZMB, GZMH). Thus, having a higher proportion of NK cells in the blood may be protective by increasing the proportions of cells with cytotoxic capabilities. Importantly, our analysis focused on transcriptome data. These findings should be further confirmed at the protein level.

The role of KLRD1 (CD94) in influenza susceptibility cannot be fully understood without considering which NKG2 family members are involved. Although bulk transcriptomic data cannot definitively answer this question, we correlated expression of KLRD1 with genes encoding NKG2 family members known to form dimers with CD94: KLRC1, KLRC2, and KLRC3. Only KLRC3, which encodes two poorly understood isoforms, NKG2E and NKG2H, correlated with KLRD1 expression at baseline. In mice, the CD94/NKG2E receptor complex is critical for recognizing and clearing mousepox infection [32]. Orbelyan and colleagues have shown that while human NKG2E has functional signaling domains and can form a complex with CD94 and DAP12, CD94/NKG2E is located in the endoplasmic reticulum, not the plasma membrane [30]. Although studies have not yet been published to address the biological relevance of this observation, this raises the possibility that human NKG2E 


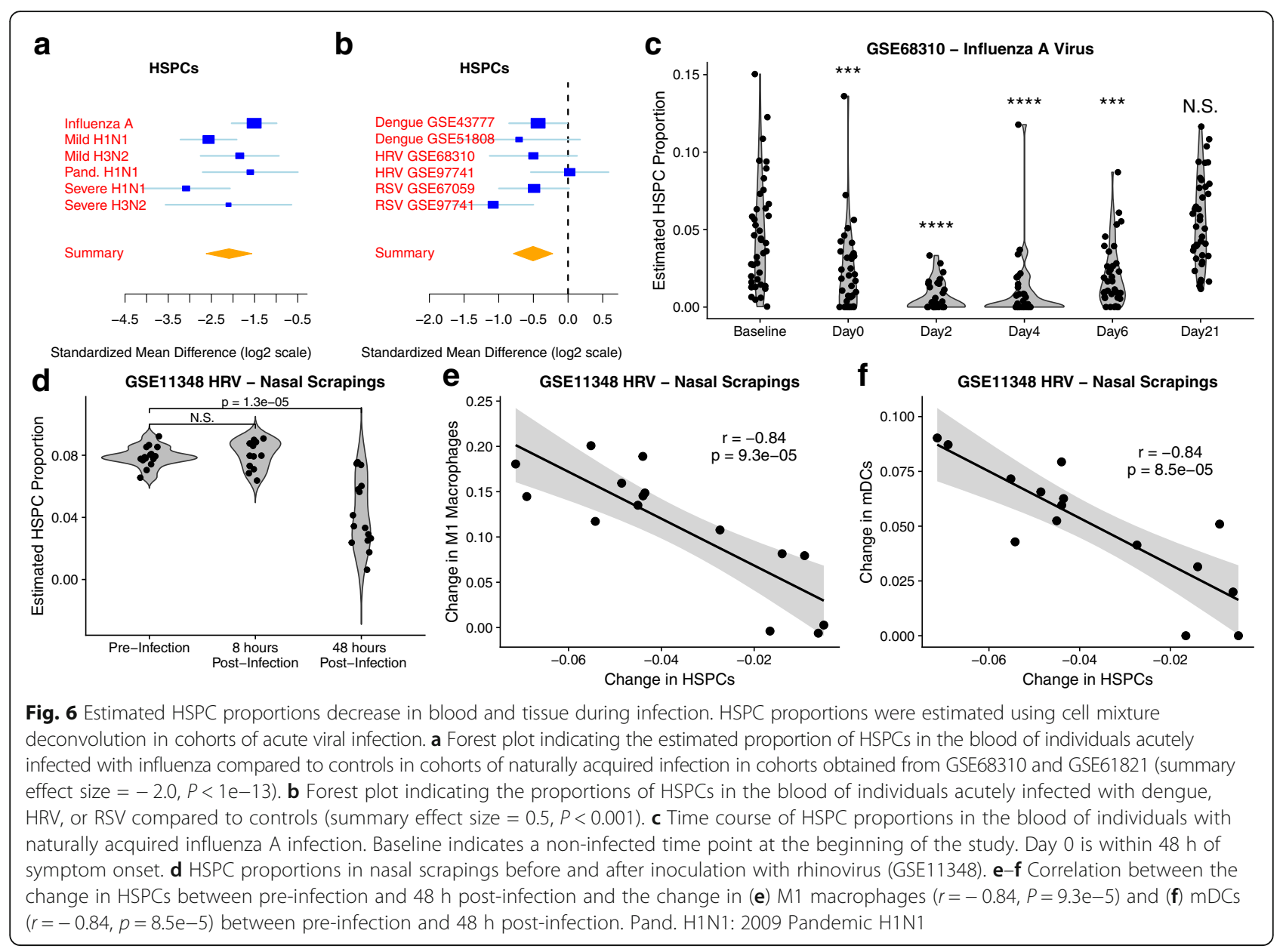

activates NK cells through an unknown intracellular pathway or inhibits NK cells by restricting the amount of DAP12 available at the cell surface. Less is known about isoform $\mathrm{NKG} 2 \mathrm{H}$, which to the best of our knowledge, has not been studied functionally in NK cells. A larger proportion of human $\mathrm{T}$ cells express NKG2H on the cell surface than NK cells, and co-crosslinking NKG2H with a NKG2H-specific monoclonal antibody prevents in vitro activation of $\mathrm{T}$ cells through an unknown mechanism [27].

These studies have interesting implications for interpreting our finding that KLRD1 is associated with influenza resistance and KLRD1 expression positively correlates with $K L R C 3$ expression in the blood. Individuals with high levels of KLRD1 (CD94) also have high levels of KLRC3 (NKG2E or NKG2H) expression. One interpretation of this observation is that there is a higher probability of forming CD94/NKG2E or CD94/NKG2H receptor complexes, based on stoichiometry. These receptor complexes could lead to influenza resistance through unidentified signaling pathways that activate NK cells. However, it is also possible that KLRC3 expression in the blood simply reflects the number of NK cells present and that KLRD1-expressing NK cells are protective against influenza using a mechanism independent of NKG2E or NKG2H signaling.

We also investigated the temporal expression of KLRD1 during influenza infection. We observed that expression of KLRD1 decreased in symptomatic shedders $48 \mathrm{~h}$ post-influenza inoculation. Therefore, we hypothesized that KLRD1-expressing cells rapidly traffic to the site of infection. However, no publicly available dataset has profiled expression from the respiratory tract of human influenza patients. Based on our previous report describing a robust common host immune response to acute respiratory viral infection including influenza, HRV, and RSV, we hypothesized that KLRD1 expression will change in the nasal epithelium of individuals infected with HRV or RSV [13]. In a HRV challenge study, KLRD1 expression in nasal scrapings $8 \mathrm{~h}$ after infection negatively correlated with symptom severity. KLRD1 expression also correlated with KLRC3 (NKG2E or $\mathrm{NKG2H)}$ and HLA-E expression. As KLRD1 and KLRC3 encode CD94/NKG2 receptor complexes, these results 
support a model where a rapid response by CD94/NKG2+ NK cells coupled with high expression of HLA-E by infected target cells leads to rapid viral clearance. Increased expression of KLRD1 and KLRC3 in nasal epithelium samples and reduced frequency of NK cells in peripheral blood samples are consistent with our hypothesis that the NK cells are actively recruited to the site of infection. Alternatively, it is possible that KLRD1 and/or KLRC3 are upregulated on NK cells in lungs of patients with respiratory viral infection or that KLRD1/KLRC3-expressing lung NK cells proliferate vigorously at that site.

Our results suggest that KLRD1 expressing NK cells may be protective against influenza. However, this is undoubtedly only one aspect of influenza susceptibility. Influenza challenge studies routinely exclude individuals with existing antibody titers to the challenge strain, meaning the results may not be directly applicable to individuals with existing $\mathrm{B}$ cell memory responses $[3,5]$. CD4+ $\mathrm{T}$ cell and CD8+ T cell cross-reactive memory responses have also been shown to affect influenza infection susceptibility and severity $[7,8]$. Hence, the role of KLRD1-expressing NK cells within broader immune system memory must be further studied.

Hematopoietic stem cells (HSCs) have the unique capacity of self-renewal [33]. HSCs differentiate into hematopoietic progenitor cells (HPCs), with varying differentiation capabilities. HSCs and HPCs are difficult to distinguish experimentally and share expression of the surface marker CD34. Thus, we use the term hematopoietic stem and progenitor cells (HSPCs) to encompass both groups. While HSPCs reside primarily in the bone marrow, it has been shown in mice that HSPCs constantly circulate from the bone marrow, through the blood, into the periphery, and finally through the lymphatic system return to the bone marrow [34]. HSPCs express Toll-like receptors (TLR), such as TLR4 and TLR2, enabling them to recognize and respond to infection [35]. In mice, TLR-stimulated HSPCs have been observed to differentiate into myeloid cell types in the periphery, including dendritic cells and macrophages [34].

Our results demonstrate that during acute viral infection, HSPC proportions decrease in the blood, which may reflect emergency myelopoiesis, a process by which hematopoiesis favors the production of myeloid cells at the expense of the lymphoid compartment to replenish myeloid cells during infection [36]. HSPC proportions may decrease in the blood during infection because HSPCs differentiate into myeloid cells in the bone marrow rather than enter circulation. Furthermore, our results demonstrate that HSPC proportions decrease in nasal scrapings upon rhinovirus challenge, and the decrease in HSPCs correlates with an increase in both M1 macrophages and mDCs. This result supports a model where human HSPCs take an active role in the immune response at the site of infection by differentiating into myeloid cells.

We identified a nonsignificant trend of reduced proportions of HSPCs in asymptomatic nonshedders prior to influenza exposure. It is possible that the asymptomatic nonshedders were protected due to a recent inflammatory event that promoted HSPC differentiation into protective M1 macrophages and mDCs. However, the likelihood of a recent inflammatory event in challenge study participants is low as subjects are often excluded from a challenge study for having had a recent flu-like illness [37]. It is also possible that the difference in HSPC proportions is due to normal variation observed in the healthy population. Further studies are needed to identify factors driving HSPC proportion variation.

Our study was limited due to our dependence on publicly available challenge study data. Arguably, the number of samples in the challenge studies used here were low. A post hoc statistical power analysis indicated we had sufficient power to detect NK cell and HSPC immune cell proportion differences [38]. We only included symptomatic shedders and asymptomatic nonshedders in our analysis. It is unclear whether our results are applicable to symptomatic nonshedders and asymptomatic shedders. Participants across all challenge studies were healthy young adults. Our results may not be applicable to children or the elderly and need to be investigated in these groups. Furthermore, we only had access to transcriptomic data. Additional studies should confirm whether symptomatic shedders have lower proportions of NK cells at baseline and whether high expression of KLRD1 in the blood directly correlates with greater numbers of CD94+ NK cells via flow cytometry.

\section{Conclusions}

In conclusion, we identified KLRD1-expressing NK cells as a novel biomarker for influenza susceptibility. We found that KLRD1 expression correlated with expression of cytotoxic granule-associated genes, suggesting that higher KLRD1 expression may correlate with increased proportions of cytotoxic immune cells. We showed that higher KLRD1 expression in the nasal epithelium $8 \mathrm{~h}$ after HRV infection was associated with reduced symptom severity. Our results imply that an early response by KLRD1-expressing NK cells may reduce symptom severity and possibly prevent influenza infection entirely. The seasonal influenza vaccine has already been shown to stimulate memory-like NK cell responses in humans [39]. Future vaccination strategies may benefit from not only 
targeting $\mathrm{B}$ cells and $\mathrm{T}$ cells but also enhancing KLRD1-expressing NK cell responses.

\section{Additional file}

Additional file 1: Table S1. Figures S1-S3; Supplemental methods. (PDF $360 \mathrm{~kb}$ )

\section{Abbreviations}

FDR: False discovery rate; GEO: Gene Expression Omnibus; HPC: Hematopoietic progenitor cell; HRV: Human rhinovirus; HSC: Hematopoietic stem cell; HSPC: Hematopoietic stem and progenitor cell; mDC: Myeloid dendritic cell; NCBI: National Center for Biotechnology Information; NK: Natural Killer; RSV: Respiratory syncytial virus; SEM: Standard error of mean

\section{Acknowledgements}

We thank Catherine Blish and Winston Haynes for the helpful discussions, and Jonathan Wosen for helping with the manuscript revision.

\section{Funding}

E.B. was supported by the Stanford Gabilan Graduate Fellowship in Science and Engineering and the Stanford Women and Sex Differences in Medicine (WSDM) Seed Grant. F.V. was supported by NIH K12 Career Award 5K12HL120001-02. P.J.U. was funded by the Donald E. and Delia B. Baxter Foundation; the Henry Gustav Floren Trust; a gift from Elizabeth F. Adler; R01 Al125197-0; and the Autoimmunity Center of Excellence grant U19-Al110491. The Autoimmunity Centers of Excellence is a research consortium supported by the National Institute of Allergy and Infectious disease (NIAID/NIH). P.K. was supported in part by grants from Bill Melinda Gates Foundation, R01 Al125197-01, 1U19Al109662, U19A1057229, U19Al090019. Funding bodies were not involved in design, interpretation, or writing of the manuscript.

\section{Availability of data and materials}

All datasets used in this study are publicly available in the Gene Expression Omnibus (GEO) database (Tables 1, 2, Additional file 1: Supplemental methods) [14].

\section{Authors' contributions}

EB and PK conceived the study. PK and FV developed the immunoStates. EB, PJU, and PK designed experiments. EB performed the data analysis under the supervision of PJU and PK. FV assisted with the analysis and interpretation of the data. EB, PJU, and PK jointly interpreted the results and wrote the manuscript with contributions from all co-authors. All authors read and approved the final manuscript.

\section{Ethics approval and consent to participate}

Not applicable.

\section{Competing interests}

The authors declare that they have no competing interests.

\section{Publisher's Note}

Springer Nature remains neutral with regard to jurisdictional claims in published maps and institutional affiliations.

\footnotetext{
Author details

${ }^{1}$ Institute for Immunity, Transplantation and Infection, Stanford University School of Medicine, Stanford, CA 94305, USA. ${ }^{2}$ Program in Immunology, Stanford University School of Medicine, Stanford 94305, CA, USA. ${ }^{3}$ Department of Medicine, Division of Biomedical Informatics Research, Stanford University School of Medicine, Stanford, CA 94305, USA. ${ }^{4}$ Department of Medicine, Division of Immunology and Rheumatology, Stanford University School of Medicine, Stanford, CA 94305, USA.
}

Received: 28 February 2018 Accepted: 24 May 2018

Published online: 14 June 2018

\section{References}

1. CDC: Disease Burden of Influenza [https://www.cdc.gov/flu/about/disease/ burden.htm].

2. Zaas AK, Chen M, Varkey J, Veldman T, Hero AO 3rd, Lucas J, Huang Y, Turner R, Gilbert A, Lambkin-Williams R, et al. Gene expression signatures diagnose influenza and other symptomatic respiratory viral infections in humans. Cell Host Microbe. 2009;6(3):207-17.

3. Woods CW, McClain MT, Chen M, Zaas AK, Nicholson BP, Varkey J, Veldman T, Kingsmore SF, Huang Y, Lambkin-Williams R, et al. A host transcriptional signature for presymptomatic detection of infection in humans exposed to influenza H1N1 or H3N2. PLoS One. 2013;8(1):e52198.

4. Muller J, Parizotto E, Antrobus R, Francis J, Bunce C, Stranks A, Nichols M, McClain M, Hill AVS, Ramasamy A, et al. Development of an objective gene expression panel as an alternative to self-reported symptom scores in human influenza challenge trials. J Transl Med. 2017;15(1):134

5. Davenport EE, Antrobus RD, Lillie PJ, Gilbert S, Knight JC. Transcriptomic profiling facilitates classification of response to influenza challenge. J Mol (Berlin). 2015;93(1):105-14.

6. Liu TY, Burke T, Park LP, Woods CW, Zaas AK, Ginsburg GS, Hero AO. An individualized predictor of health and disease using paired reference and target samples. BMC bioinformatics. 2016;17:47.

7. Wilkinson TM, Li CK, Chui CS, Huang AK, Perkins M, Liebner JC, LambkinWilliams R, Gilbert A, Oxford J, Nicholas B, et al. Preexisting influenza-specific CD4+ T cells correlate with disease protection against influenza challenge in humans. Nat Med. 2012;18(2):274-80.

8. Sridhar S, Begom S, Bermingham A, Hoschler K, Adamson W, Carman W, Bean T, Barclay W, Deeks JJ, Lalvani A. Cellular immune correlates of protection against symptomatic pandemic influenza. Nat Med. 2013;19(10): 1305-12.

9. Shen-Orr SS, Gaujoux R. Computational deconvolution: extracting cell typespecific information from heterogeneous samples. Curr Opin Immunol. 2013;25(5):571-8.

10. Inkeles MS, Teles RM, Pouldar D, Andrade PR, Madigan CA, Lopez D, Ambrose M, Noursadeghi M, Sarno EN, Rea TH, et al. Cell-type deconvolution with immune pathways identifies gene networks of host defense and immunopathology in leprosy. JCl insight. 2016;1(15):e88843.

11. Gentles AJ, Newman AM, Liu CL, Bratman SV, Feng W, Kim D, Nair VS, Xu Y, Khuong A, Hoang CD, et al. The prognostic landscape of genes and infiltrating immune cells across human cancers. Nat Med. 2015;21(8):938-45.

12. Vallania F, Tam A, Lofgren S, Schaffert S, Azad TD, Bongen E, Alsup M, Alonso M, Davis M, Engleman E, et al. Leveraging heterogeneity across multiple data sets increases accuracy of cell-mixture deconvolution and reduces biological and technical biases. In: bioRxiv; 2017.

13. Andres-Terre M, McGuire Helen M, Pouliot Y, Bongen E, Sweeney Timothy E, Tato Cristina M, Khatri P. Integrated, multi-cohort analysis identifies conserved transcriptional signatures across multiple respiratory viruses. Immunity. 2015:43(6):1199-211.

14. Edgar R, Domrachev M, Lash AE. Gene Expression Omnibus: NCBI gene expression and hybridization array data repository. Nucleic Acids Res. 2002; 30(1):207-10.

15. Haynes WA, Vallania F, Liu C, Bongen E, Tomczak A, Andres-Terre M, Lofgren S, Tam A, Deisseroth CA, Li MD, et al. Empowering multi-cohort gene expression analysis to increase reproducibility. Pac Symp Biocomput. 2017; 22:144-53.

16. Lofgren S, Hinchcliff M, Carns M, Wood T, Aren K, Arroyo E, Cheung P, Kuo A, Valenzuela A, Haemel A, et al. Integrated, multicohort analysis of systemic sclerosis identifies robust transcriptional signature of disease severity. JCI insight. 2016;1(21):e89073.

17. Sweeney TE, Haynes WA, Vallania F, loannidis JP, Khatri P. Methods to increase reproducibility in differential gene expression via meta-analysis. Nucleic Acids Res. 2017:45(1):e1-e1.

18. Benjamini $Y$, Hochberg $Y$. Controlling the false discovery rate: a practical and powerful approach to multiple testing. J R Statist Soc. 1995;B57:289-300.

19. Martinet L, Smyth MJ. Balancing natural killer cell activation through paired receptors. Nat Rev Immunol. 2015:15(4):243-54.

20. Kumar D, Hosse J, von Toerne C, Noessner E, Nelson PJ. JNK MAPK pathway regulates constitutive transcription of CCL5 by human NK cells through SP1. J Immunol. 2009;182(2):1011-20. 
21. Lam VC, Lanier LL. NK cells in host responses to viral infections. Curr Opin Immunol. 2017:44:43-51.

22. Proud D, Turner RB, Winther B, Wiehler S, Tiesman JP, Reichling TD, Juhlin KD, Fulmer AW, Ho BY, Walanski AA, et al. Gene expression profiles during in vivo human rhinovirus infection: insights into the host response. Am J Respir Crit Care Med. 2008;178(9):962-8.

23. Do LAH, Pellet J, van Doorn HR, Tran AT, Nguyen BH, Tran TTL, Tran QH, Vo $\mathrm{QB}$, Tran Dac NA, Trinh HN, et al. Host transcription profile in nasal epithelium and whole blood of hospitalized children under 2 years of age with respiratory syncytial virus infection. J Infect Dis. 2017;217(1):134-46.

24. Zhai Y, Franco LM, Atmar RL, Quarles JM, Arden N, Bucasas KL, Wells JM, Nino D, Wang X, Zapata GE, et al. Host transcriptional response to influenza and other acute respiratory viral infections-a prospective cohort study. PLoS Pathog. 2015;11(6):e1004869.

25. Lieto LD, Maasho K, West D, Borrego F, Coligan JE. The human CD94 gene encodes multiple, expressible transcripts including a new partner of NKG2A B. Genes Immun. 2006;7(1):36-43.

26. Orbelyan GA, Tang F, Sally B, Solus J, Meresse B, Ciszewski C, Grenier JC, Barreiro LB, Lanier LL, Jabri B. Human NKG2E is expressed and forms an intracytoplasmic complex with CD94 and DAP12. J Immunol. 2014;193(2): 610-6.

27. Dukovska D, Fernandez-Soto D, Vales-Gomez M, Reyburn HT. NKG2Hexpressing T cells negatively regulate immune responses. Front Immunol. 2018;9:390.

28. Fang $M$, Orr MT, Spee $P$, Egebjerg T, Lanier LL, Sigal LJ. CD94 is essential for NK cell-mediated resistance to a lethal viral disease. Immunity. 2011;34(4): 579-89.

29. Shin DL, Pandey AK, Ziebarth JD, Mulligan MK, Williams RW, Geffers R, Hatesuer B, Schughart K, Wilk E. Segregation of a spontaneous KIrd1 (CD94) mutation in DBA/2 mouse substrains. G3 (Bethesda). 2014;5(2):235-9.

30. Orr MT, Wu J, Fang M, Sigal L, Spee P, Egebjerg T, Dissen E, Fossum S, Phillips JH, Lanier LL. Development and function of CD94-deficient natural killer cells. PLoS One. 2010;5(12):e15184.

31. Abdul-Careem MF, Mian MF, Yue G, Gillgrass A, Chenoweth MJ, Barra NG, Chew MV, Chan T, Al-Garawi AA, Jordana M, et al. Critical role of natural killer cells in lung immunopathology during influenza infection in mice. J Infect Dis. 2012;206(2):167-77.

32. Ge MQ, Ho AW, Tang Y, Wong KH, Chua BY, Gasser S, Kemeny DM. NK cells regulate $C D 8+T$ cell priming and dendritic cell migration during influenza A infection by IFN-gamma and perforin-dependent mechanisms. J Immunol. 2012;189(5):2099-109.

33. Mazo IB, Massberg S, von Andrian UH. Hematopoietic stem and progenitor cell trafficking. Trends Immunol. 2011;32(10):493-503.

34. Massberg S, Schaerli P, Knezevic-Maramica I, Kollnberger M, Tubo N, Moseman EA, Huff IV, Junt T, Wagers AJ, Mazo IB, et al. Immunosurveillance by hematopoietic progenitor cells trafficking through blood, lymph, and peripheral tissues. Cell. 2007;131(5):994-1008.

35. Nagai Y, Garrett KP, Ohta S, Bahrun U, Kouro T, Akira S, Takatsu K, Kincade PW. Toll-like receptors on hematopoietic progenitor cells stimulate innate immune system replenishment. Immunity. 2006;24(6):801-12.

36. Takizawa H, Boettcher S, Manz MG. Demand-adapted regulation of early hematopoiesis in infection and inflammation. Blood. 2012;119(13):29913002.

37. Lillie PJ, Berthoud TK, Powell TJ, Lambe T, Mullarkey C, Spencer AJ, Hamill M, Peng Y, Blais ME, Duncan CJ, et al. Preliminary assessment of the efficacy of a T-cell-based influenza vaccine, MVA-NP+M1, in humans. Clin Infect Dis. 2012;55(1):19-25.

38. Valentine JC, Pigott TD, Rothstein HR. How many studies do you need?:a primer on statistical power for meta-analysis. J Educ Behav Stat. 2010;35(2): 215-47.

39. Dou Y, Fu B, Sun R, Li W, Hu W, Tian Z, Wei H. Influenza vaccine induces intracellular immune memory of human NK cells. PLoS One. 2015;10(3): e0121258.

40. Hoang LT, Tolfvenstam T, Ooi EE, Khor CC, Naim AN, Ho EX, Ong SH, Wertheim HF, Fox A, Van Vinh Nguyen C, et al. Patient-based transcriptomewide analysis identify interferon and ubiquination pathways as potential predictors of influenza A disease severity. PLoS One. 2014;9(11):e111640.

41. Sun P, Garcia J, Comach G, Vahey MT, Wang Z, Forshey BM, Morrison AC, Sierra G, Bazan I, Rocha C, et al. Sequential waves of gene expression in patients with clinically defined dengue illnesses reveal subtle disease phases and predict disease severity. PLoS Negl Trop Dis. 2013;7(7):e2298.
42. Kwissa M, Nakaya HI, Onlamoon N, Wrammert J, Villinger F, Perng GC, Yoksan S, Pattanapanyasat K, Chokephaibulkit K, Ahmed R, et al. Dengue virus infection induces expansion of a CD14(+)CD16(+) monocyte population that stimulates plasmablast differentiation. Cell Host Microbe. 2014;16(1):115-27.

43. Heinonen S, Jartti T, Garcia C, Oliva S, Smitherman C, Anguiano E, de Steenhuijsen Piters WA, Vuorinen T, Ruuskanen O, Dimo B, et al. Rhinovirus detection in symptomatic and asymptomatic children: value of host transcriptome analysis. Am J Respir Crit Care Med. 2016;193(7):772-82.

\section{Ready to submit your research? Choose BMC and benefit from:}

- fast, convenient online submission

- thorough peer review by experienced researchers in your field

- rapid publication on acceptance

- support for research data, including large and complex data types

- gold Open Access which fosters wider collaboration and increased citations

- maximum visibility for your research: over $100 \mathrm{M}$ website views per year

At BMC, research is always in progress.

Learn more biomedcentral.com/submissions 